\title{
Differential Mobility of Breast Cancer Cells and Normal Breast Epithelial Cells Under DC Electrophoresis and Electroosmosis
}

\author{
DIGANTA DUTTA ${ }^{1}$, CHRISTOPHER RUSSELL $^{1}$, JINHYUN KIM $^{1}$ and SURABHI CHANDRA ${ }^{2}$ \\ ${ }^{1}$ Department of Physics and Astronomy, University of Nebraska-Kearney, Kearney, NE, U.S.A.; \\ ${ }^{2}$ Department of Biology, University of Nebraska-Kearney, Kearney, NE, U.S.A.
}

\begin{abstract}
Background/Aim: Breast cancer has shown improved prognosis over the years, yet due to different types of breast cancer and different phenotypes of breast cancer cells, it is essential to have an accurate diagnostic procedure. Materials and Methods: A unique methodology employing direct current $(D C)$ electrophoresis and electroosmosis in fabricated microfluidic devices was used. This phenomenon was used to discern breast cancer cells from normal breast epithelial cells based on their electrophoretic mobility and electroosmosis. Results: Breast cancer cells (MDA-MB-231) rapidly covered the wound in scratch assay compared to mammary epithelial cells (MCF-10A). However, under DC electrophoresis and electroosmosis MCF-10A cells were observed to move at higher velocities compared to MDA-MB231 cells under similar voltages. Conclusion: Our study provides an economical and efficient method of studying electrophoretic and electrokinetic phenomenon of different cell types that can be used to the advantage of detecting and monitoring cancer progression.
\end{abstract}

Cancer is the second leading cause of death worldwide, and has a heavy economic burden ( $\$ 1.17$ trillion, 2010) on the society (1). Breast cancer is the most commonly diagnosed cancer and is the second leading cause of cancer-related death among women. Although the incidence of breast cancer has considerably declined and medical advancement has increased life expectancy, risk factors for cancer including aging and obesity are on a rise, and thus measures need to be taken for early diagnosis and treatment.

Microfabrication at the cellular level is the new technological tool for detecting and treating cancer. Cancer

Correspondence to: Surabhi Chandra, Ph.D., 2401 W. 11th Ave, BHS335, Department of Biology, University of Nebraska-Kearney, Kearney, NE 68849, U.S.A. Tel: +1 3088658661, Fax: +1 3088658045, e-mail: chandras2@unk.edu

Key Words: Breast cancer cells, DC electrophoresis, electroosmosis, microfluidics, cell mobility. cells possess unique characteristics: growth at low oxygen, less negative membrane potential, lower membrane capacitance and cytoplasmic conductivity, and fermentative metabolism compared to normal healthy cells (2). These properties are being exploited to discern cancer cells from normal healthy cells in a population. In this article, we report for the first time a new feature exploiting differential velocity of cells (tumor versus normal) under electric field using the phenomenon of direct current (DC) electroosmosis and electrophoresis.

Lab-on-a-chip-based devices are being used to analyze chemicals and for manipulation of biological cells and tissues (3-6). Electroosmosis is defined as the motion of liquid relative to a charged surface under the influence of an electric field (7). The two main classifications of this process include DC and alternating current (AC) electroosmosis.

As shown in Figure 1a the negatively charged walls along the channel allow electroosmosis to occur. This process is due to the formation of an electric double layer (EDL) from potassium chloride solution that comes in contact with a charged surface. Electroosmotic velocity can be measured in microchannels using Helmholtz-Smoluchowski approximation for thin EDL (Equation 1).

$$
u_{E O}=-\frac{\varepsilon_{f} \varepsilon_{0} \zeta}{\mu} E \mathrm{x} \quad \text { Equation } 1
$$

$\mathrm{u}_{\mathrm{EO}}$ is the Helmholtz-Smoluchowski electroosmotic velocity, $\zeta$ is the zeta potential, $\varepsilon_{0}$ is the absolute permittivity in the vacuum, $\varepsilon$ is permittivity of the liquid, $\varepsilon_{f}$ is the relative permittivity of the fluid, $\mu$ is the dynamic viscosity of the fluid, and $E_{x}$ is the electric field.

Similar to electroosmosis, electrophoresis describes the motion of a charged particle relative to its surrounding liquid (8) (Figure 1b). The separation and behavior of particles during electrophoresis is a result of varying velocities (v), which is a product of particle's mobility $(\mu)$ and applied voltage (E) (Equation 2).

$$
\mathrm{v}=\mu \mathrm{E}
$$

Equation 2 

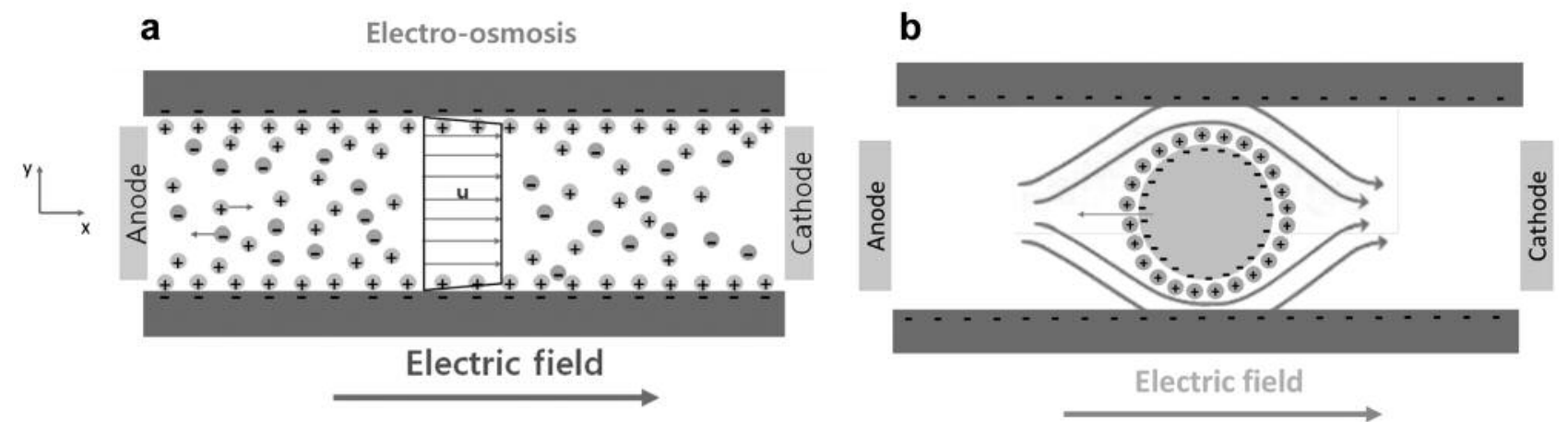

Figure 1. Electroosmosis and electrophoresis phenomenon in microchannel (12, 13).

In this paper, both electrophoresis and electroosmosis were utilized to understand the mobility of cells when subjected to voltage variations. In biological systems, cancer cells show increased movement (metastasis) compared to differentiated tissue cells, however, voltage application can alter the behavior of both cell types due to variations in cellular morphologies.

\section{Materials and Methods}

Cell culture. MDA-MB-231 (breast cancer cells), and MCF-10A (non-tumorigenic mammary epithelial cells) were obtained from the American Type Culture Collection (Manassas, VA, USA). MDAMB-231 cells were grown in DMEM/F12 media supplemented with $5 \%$ fetal bovine serum, whereas MCF-10A cells were grown in DMEM/F12 media supplemented with $5 \%$ horse serum, $20 \mathrm{ng} / \mathrm{ml}$ epidermal growth factor, $0.5 \mu \mathrm{g} / \mathrm{ml}$ hydrocortisone, $100 \mathrm{ng} / \mathrm{ml}$ cholera toxin, and $10 \mu \mathrm{g} / \mathrm{ml}$ insulin.

Scratch wound healing assay. Cells were grown in 6-well plates to complete confluency after overnight incubation. A scratch was made in the most confluent zone using a sterile $1 \mathrm{ml}$ pipet tip, floating cells were washed once with ice-cold PBS and incubated with media. Phase-contrast images were taken at 0,24 and $48 \mathrm{~h}$ to analyze gap closure.

Construction of microfluidic device. Glass slides were sonicated with $1 \mathrm{M} \mathrm{KOH} \mathrm{(Acros} \mathrm{Organics,} \mathrm{NJ,} \mathrm{USA)} \mathrm{(15} \mathrm{min),} \mathrm{rinsed} \mathrm{with}$ DI water (2 to $3 \mathrm{~min})$, sonicated with acetone $(15 \mathrm{~min})$, rinsed with DI water (2 to $3 \mathrm{~min}$ ), sonicated with isopropanol (Fisher Science Education, Pittsburgh, PA, USA) (15 min), and then rinsed with isopropanol and DI water (2 to $3 \mathrm{~min}$ ). Finally, sample surfaces were sonicated with DI water following a 15-min rinse with acetone, ethanol and DI water. Samples were dried with air gun and then put above hot plate at $120^{\circ} \mathrm{C}$ for $3 \mathrm{~min}$. These sample surfaces were stored in clean boxes to avoid dust.

Microfluidic device consisted of a microchannel with two reservoirs on opposing sides of the channel (Figure 2). A clean razor blade was used to cut a thin strip of clear tape into the desired channel shape. This strip was placed into a petri dish. A prepolymer and curing agent with a 10:1 ratio was poured into the petri dish.

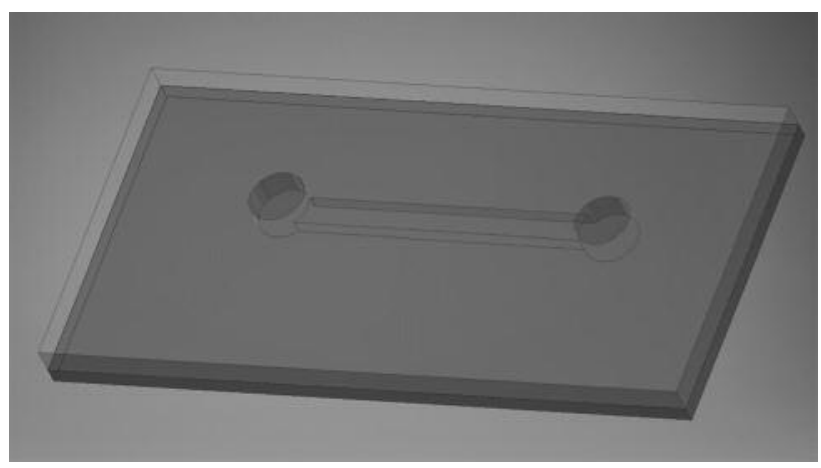

Figure 2. Schematic diagram of the microfluidic device.

The resultant polydimethylsiloxane (PDMS) was left to cure for 36 $\mathrm{h}$ at room temperature. After completion, the cured PDMS along with the microfluidics channel was carefully removed from the petri dish using a clean razor blade. Before adhering PDMS to the glass slide, the two reservoirs were punched into either side of the microfluidics channel. Deionized water was used to clear the reservoirs, and the entire slide was cleaned with a corona discharge. Following cleaning process PDMS was gently placed upon the clean glass slide.

Microfluidic measurements. A charge-couple device, or CCD camera, was used to capture the location of cells at a given time (every ten milliseconds) (Figure 3). These images were used to conduct velocity field measuremtents through 2-D micro-PIV image acquisition to target specific cells. A MATLAB code was used to analyze the images. Microscope magnification of $4 \times$ was used to analyze the desired vector field of the fluid.

\section{Results}

A relatively steady positive relationship between maximum velocity and applied voltage was observed throughout the interval (Figures 4 and 5). A spike in velocity was shown 


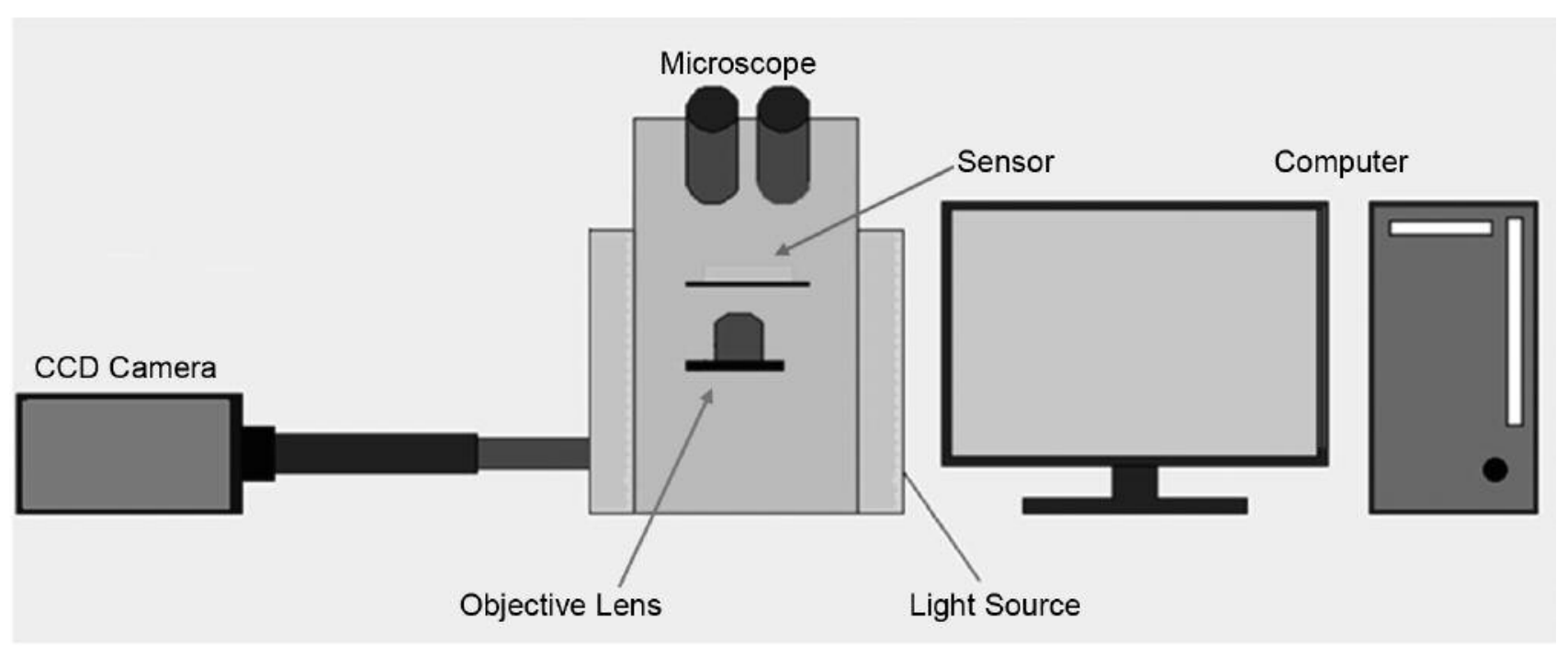

Figure 3. Schematic diagram of the experimental set up.

between $3 \mathrm{~V}$ and $3.5 \mathrm{~V}$ as well as between $4.5 \mathrm{~V}$ and $5 \mathrm{~V}$ for MDA-MB-231 cells. The maximum velocity at any voltage was much lower than the corresponding velocity for MCF10A cell. MCF-10A cells were shown to have a loose exponential relationship with velocity and applied voltages (Figure 5). At lower voltages ( $1 \mathrm{~V}$ to $4 \mathrm{~V})$, the increase in maximum velocity was rather gradual, however, there was nearly a $100 \mathrm{~mm} / \mathrm{sec}$ increase in the recorded velocities from $4 \mathrm{~V}$ to $4.5 \mathrm{~V}$. Between $4.5 \mathrm{~V}$ and $5 \mathrm{~V}$, the velocity continued to increase at a smaller rate and changed by roughly $33.67 \mathrm{~mm} / \mathrm{sec}$.

The electro kinetic process gives a range of applied voltages spanning between $1 \mathrm{~V}$ and $5 \mathrm{~V}$. A relatively steady positive relationship between maximum velocity and applied voltage was observed throughout this interval (Figures 4 and 5). A spike in velocity was noted between $3 \mathrm{~V}$ and $3.5 \mathrm{~V}$ as well as between $4.5 \mathrm{~V}$ and $5 \mathrm{~V}$ (Figure 4 ). The relationship between maximum velocity and applied voltage was relatively similar to that of MCF-10A cell (Figure 5). However, the maximum velocity at $1 \mathrm{~V}$ of MDA-MB-231 was significantly lower than MCF-10A cell at roughly 2.919 $(\mathrm{mm} / \mathrm{s})$. This trend continued throughout the various applied voltages. At $5 \mathrm{~V}$, the largest maximum velocity throughout the given interval was found to be $61.05532(\mathrm{~mm} / \mathrm{s})$ (Figure 4). This value was much lower than the velocity for the MCF-10A cell which was $199.0956(\mathrm{~mm} / \mathrm{s})$ at the same voltage (Figure 5).

MCF-10A cells were shown to have a loose exponential relationship with velocity and applied voltages (Figure 5). At lower voltages, from $1 \mathrm{~V}$ to $4 \mathrm{~V}$, the increase in maximum velocity was rather gradual. The average change in velocity between these two voltages was roughly $19.33(\mathrm{~mm} / \mathrm{s}) / \mathrm{V}$. This trend stopped once the applied voltage surpasses $4 \mathrm{~V}$. There was nearly a $100 \mathrm{~mm} / \mathrm{s}$ increase between the recorded velocities at $4 \mathrm{~V}$ and $4.5 \mathrm{~V}$. Interestingly, this jump was not observed anywhere else between $1 \mathrm{~V}$ and $5 \mathrm{~V}$ of the recorded data. Between $4.5 \mathrm{~V}$ and $5 \mathrm{~V}$, the change in velocity came down to roughly $33.67 \mathrm{~mm} / \mathrm{s}$. An undisputed trend throughout the data is that as the applied voltage is increased the maximum velocity of the fluid increases as well.

Cellular mobility in the absence of applied voltage was evaluated using scratch wound healing assay. Clear zone in the scratch wound assay represents wound created in a confluent cell layer using a micropipette tip (Figure 6). Cell movement was observed in MDA-MB-231 cells within $24 \mathrm{~h}$ of creating the wound which was completely covered at about $48 \mathrm{~h}$. MCF-10A cells showed no movement towards the gap and the wound remained intact.

\section{Discussion}

Breast cancer, in particular, has received great attention as it is the leading cause of cancer-related mortality in women and also has a genetic predisposition. Phenotypically cancer cells display unique properties such as uncontrolled proliferation, immortality, invasiveness, and metastasis (9). The most common and rapid method for detection of cancer is using a biopsy but it is invasive. Other methods used to monitor progression of cancer including imaging techniques (CT, MRI, and sonography), and biomarker analysis (ELISA, PCR) are time consuming and expensive (10). Microfluidic devices in this context have been designed for 

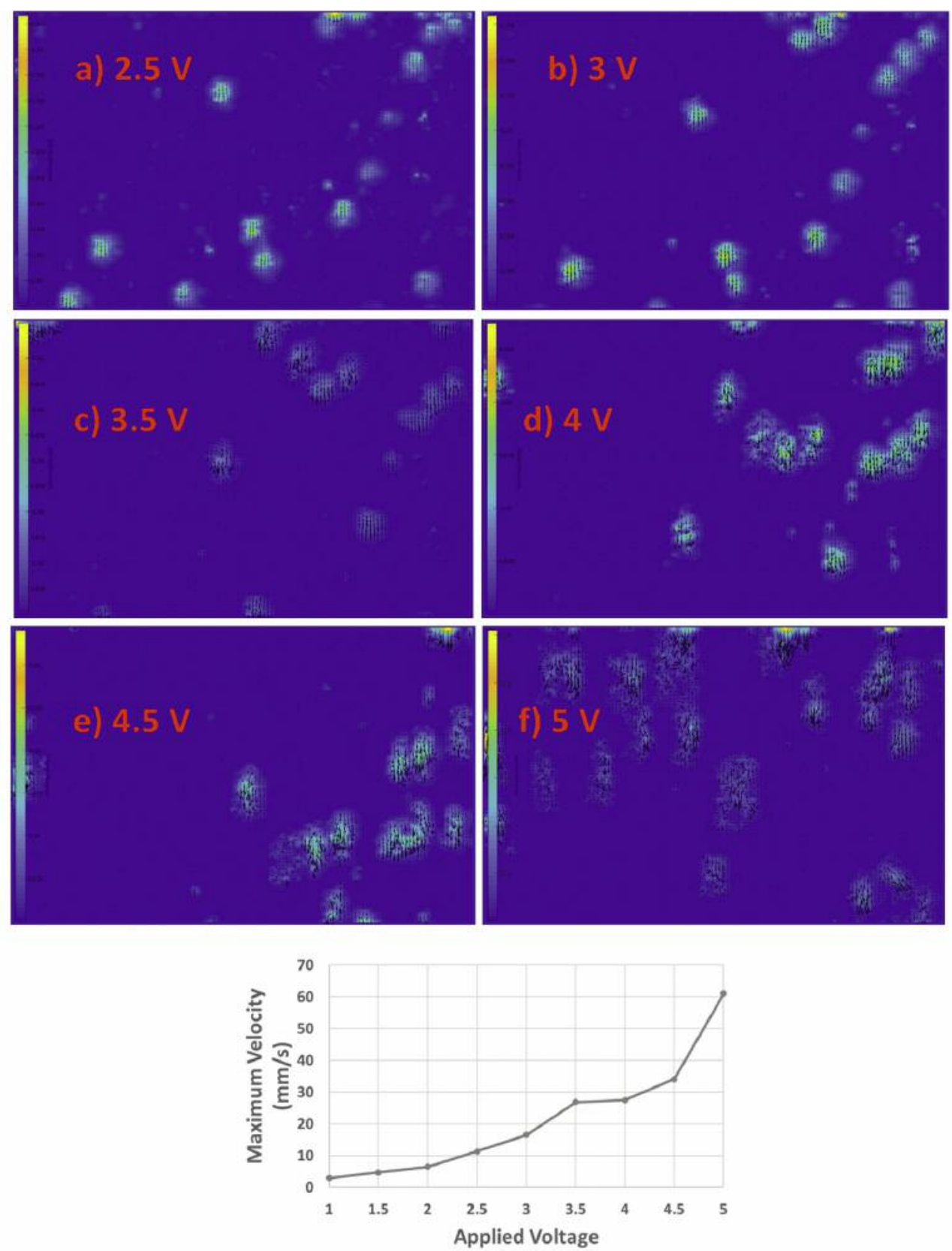

Figure 4. Velocity vector at different applied voltages (a-f, top) and relationship of maximum velocity in MDA-MB-231 cancer cells with applied voltages in microfluidic device (bottom).

multiple tumor biomarker analysis in a single setting using electro-chemiluminescence, power-free miRNA analysis, and label-free size-based isolation in pancreatic cancer, gastric cancer, and gastritis (10). In this paper, we reported a novel methodology of discerning breast cancer cells from normal breast epithelial cells using principles of electroosmosis and electrophoresis in a microfluidic device.
Triple-negative advanced-stage breast cancer cell line, MDA-MB-231, displays similar genetic profile as nontumorigenic mammary epithelial cells, MCF-10A. However, invasive cell lines (MDA-MB-231) exhibit higher spreading rate and altered cytoskeletal arrangements compared to noninvasive cell lines (MCF-10A) regardless of their genetic profile (11). This has been attributed to differential expression 

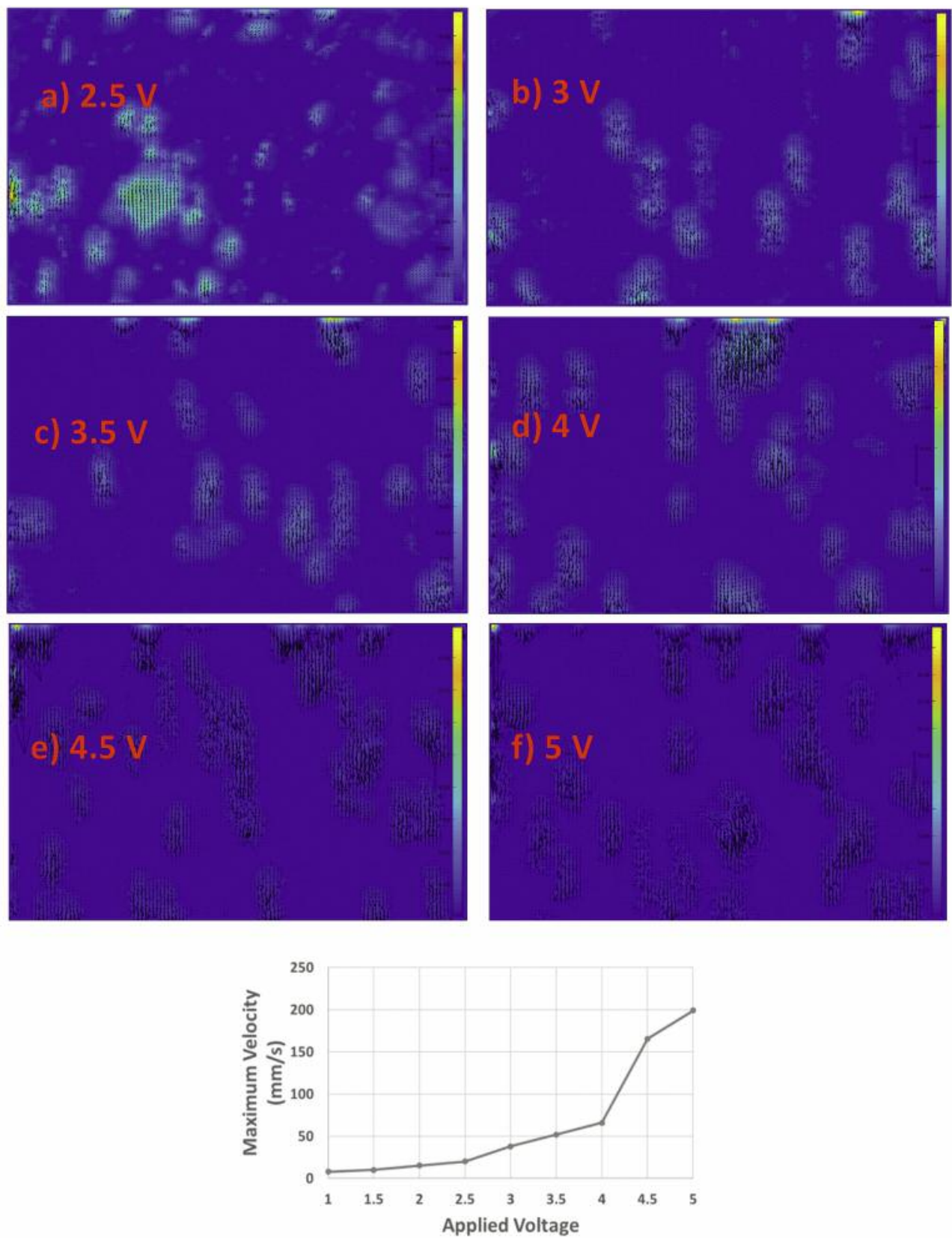

Figure 5. Velocity vector at different applied voltages (a-f, top) and relationship of maximum velocity in MCF-10A cells with applied voltages in microfluidic device (bottom).

of structural proteins. Our results showed that cancer cells rapidly covered scratch wound in cell culture, indicative of metastasis, though MCF-10A cells showed no movement towards the area of wound despite longer incubation. Electrophoretic cell movement, in contrast, displayed higher mobility for normal cells compared to cancer cells for similar voltages. In a study focused on separating cells by electrokinetics in biological fluids using AC voltages, it was shown that while cancer cells remained closer to the top of the center electrode, non-tumorigenic white blood cells due to their smaller size and lesser polarizability, showed fluidic movement away from the electrode (12).

The main goal of this work was to fabricate very simple fluidic devices and generate both DC electroosmosis and 


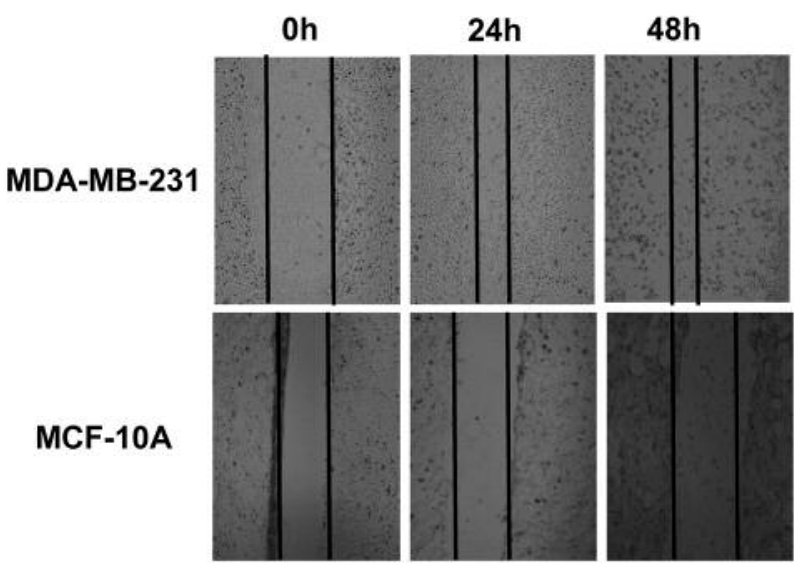

Figure 6. Scratch wound healing over $48 \mathrm{~h}$ in $M D A-M B-231$ and MCF-10A cells.

electrophoresis transport phenomena. It was demonstrated that the velocity of both cell lines increased with increasing voltage, and the velocity was much higher for normal mammary epithelial cells (MCF-10A) compared to cancer cells (MDA-MB-231), which suggests a possible structural difference that will be further investigated.

\section{Conclusion}

This paper provides a unique and cost-efficient methodology of understanding motility behavior of cancer cells and normal epithelial cells under DC electrophoresis and electroosmosis. This process can be used to array a wide range of cancer cells based on their variable movement under the influence of applied voltage.

\section{Acknowledgements}

This study was funded by UNK Research Services Council Collaborative Grant awarded to DD and SC. Instrumentation and tissue culture facility were supported by a grant from the National Institute for General Medical Science (NIGMS) (5P20GM103427), a component of the National Institutes of Health (NIH).

\section{References}

1 WHO: World Health Organization Health Sheet 2018 [Available from: http://www.who.int/news-room/fact-sheets/detail/cancer.

2 Sundararajan R: Electroporation-based therapies for cancer: From basics to clinical applications, Woodhead Publishing, pp. 306, 2014.

3 Cui H, Horiuchi K, Dutta P and Ivory CF: Isoelectric Focusing in a Poly(dimethylsiloxane) Microfluidic Chip. Anal Chem 77(5): 1303-1309, 2005.

4 Cui H, Horiuchi K, Dutta P and Ivory CF: Multistage isoelectric focusing in a polymeric microfluidic chip. Anal Chem $77(24)$ : 7878-7886, 2005.

5 Matteucci M, Heiskanen A, Zor K, Emneus J and Taboryski R: Comparison of ultrasonic welding and thermal bonding for the integration of thin film metal electrodes in injection molded polymeric lab-on-chip systems for electrochemistry. Sensors 16(11), 2016.

6 Mondal K, Ali MA, Srivastava S, Malhotra BD and Sharma A: Electrospun functional micro/nanochannels embedded in porous carbon electrodes for microfluidic biosensing. Sensors and Actuators B: Chemical 229: 82-91, 2016.

7 Hossan MR, Dutta D, Islam N and Dutta P: Review: Electric field driven pumping in microfluidic device. Electrophoresis 39(5-6): 702-731, 2018.

8 Fritsch RJ and Krause I: Electrophoresis. In: Caballero B, editor. Encyclopedia of Food Sciences and Nutrition. Second Edition, Oxford Academic Press, 2003.

9 Poli V, Fagnocchi L and Zippo A: Tumorigenic cell reprogramming and cancer plasticity: interplay between signaling, microenvironment, and epigenetics. Stem Cells Int 2018: 4598195, 2018.

10 Wu J, Dong M, Santos S, Rigatto C, Liu Y and Lin F: Lab-ona-chip platforms for detection of cardiovascular disease and cancer biomarkers. Sensors 17(12): pii: E2934, 2017.

11 Chiotaki R, Polioudaki H and Theodoropoulos PA: Differential nuclear shape dynamics of invasive andnon-invasive breast cancer cells are associated with actin cytoskeleton organization and stability. Biochem Cell Biol 92(4): 287-295, 2014.

12 Gao J, Riahi R, Sin ML, Zhang S and Wong PK: Electrokinetic focusing and separation of mammalian cells in conductive biological fluids. Analyst 137(22): 5215-5221, 2012.

13 Patel RG: Time resolved electrophoretic and electroosmotic velocities: Rutgers University-Graduate School, The State University of New Jersey, New Brunswick, NJ, USA, 2013.

Received August 16, 2018

Revised September 2, 2018

Accepted September 4, 2018 